\title{
Facial and dental alterations according to the breathing pattern
}

\author{
Luciana Borges RETAMOSO', Luégya Amorin Henriques KNOP², Odilon GUARIZA FILHO³, \\ Orlando Motohiro TANAKA ${ }^{4}$ \\ 1- DDS, MSc, Doctor of Science student, Pontifical Catholic University of Rio Grande do Sul, Porto Alegre, RS, Brazil. \\ 2- DDS, MSc, Pontifical Catholic University of Paraná, Curitiba, PR, Brazil. \\ 3- DDS, MSc, PhD, Associate Professor, Pontifical Catholic University of Paraná, Curitiba, Brazil. \\ 4- DDS, MSc, PhD, Senior Professor and Chair, Pontifical Catholic University of Paraná, Curitiba, Brazil.
}

Corresponding address: Orlando Motohiro Tanaka - Av. Mal Deodoro, 630, 1703 - 80010-912 - Curitiba - PR Brazil - Phone: +55-42 3323-2042 - Fax: +5541-3324-8768 - e-mail: tanakaom@gmail.com

Received: July 18, 2009 - Accepted: May 18, 2010

\section{ABSTRACT}

There is controversy in the literature about possible interaction of the respiratory
mode with the facial and dental structures. Objectives: The aim of this study was to
perform a longitudinal assessment of the changes in facial and dental structures in Angle's
Class II, division 1 malocclusion individuals, divided according to the respiratory pattern
(predominantly nasal or mouth), at two distinct moments of craniofacial development.
Material and Methods: Pogonium and nose measurements were made on the lateral
cephalometric tracings (LS'-Pog', LS'-B', B'-Pog', Pog'-PogTeg', Line NB, Pog-NB, N'-Prn,
Prn-NPog, N-Prn-Sn, Prn-Sn-LS). Dental measurements were made on the plaster models
(distances between the tips of the canine cusps and the tips of mesial cusps of the first
molars) of 40 individuals aged 10 to 14 years (moment 1 ) and 13 to 16 years (moment
2), 23 being nose breathers (NB) and 17 being predominantly mouth breathers (MB).
Results: The Student's-t test and two-way ANOVA with repeated measures were applied to
indicate differences between the mean values of these variables according to the moments
and/or respiratory mode. Conclusions: There were alterations in the facial measurements,
without interference of the breathing pattern. However, the breathing pattern influenced
dental alterations.

Key words: Angle Class II. Nose. Breathing. Dental arch. Chin.

\section{INTRODUCTION}

The size and shape of the dental arches have considerable implications for the diagnosis and orthodontic treatment plan, affecting the available space, esthetics and stability of dentition ${ }^{17}$, and according to Knott ${ }^{15}$ (1972), the size and shape of the dental arches are not static. The width of the dental arches is a dimension that alters significantly during the development of dentition, and can be studied by means of the intercanine and intermolar distances ${ }^{10}$.

The individual's soft profile is the result of changes that occur in the skeletal and soft tissues of the facial structures, and the inter-relationship between the components of the soft tissues of the face, such as the nose, lip and the chin or pogonium, change during growth and throughout the course of orthodontic treatment. Therefore, it is important to understand the normal growth trends of this structures ${ }^{19}$.

The nose is the most dominant factor of all the elements of the profile, yet it still receives little attention in orthodontic analysis, although facial analyses, such as those of Steiner ${ }^{24}$ (1959), Ricketts $^{21}$ (1957), Hambleton ${ }^{11}$ (1964) and Chaconas $^{6}$ (1969) used the nose as a point of reference, or as another element of information.

With regard to the influence of the respiratory mode on the morphology of the dentoskeletal complex, there are many publications in the literature, but there is still a great deal of disagreement among the authors. Some report that obstruction of nasal respiration alters facial 
growth and development ${ }^{12}$; others affirm the altered growth of the dentofacial complex results from environmental and genetic factors ${ }^{28}$.

The majority of studies found in the literature discuss the alterations in the intercanine and intermolar distance in individuals treated and not treated with the biomechanical resources of Orthodontics, and among cases with and without extractions, and in the contention phase. Longitudinal studies in individuals not orthodontically treated are scarce s,3,8,27. $^{1,}$

The present study intended to assess whether the respiratory mode influences the dental intercanine and intermolar distances - and facial measurements, by analysis of the pogonium and nose.

\section{MATERIAL AND METHODS}

The sample was composed of lateral teleradiographs of 40 individuals with Angle's Class II, division 1 malocclusion, 23 being predominantly nose breathers (RN) and 17 predominantly mouth breathers (RB), aged between 10 years, 9 months and 14 years (moment 1 ), and between 13 years, 4 months and 16 years, 6 months (moment 2 ).

The breathing pattern classification was performed multidisciplinary, including clinical evaluation of lips protrusion or retrusion by orthodontist, questionnaire filled out by parents concerning the breathing habits as well as otolaryngology and speech and audiology evaluations. From these assessments, weights were assigned points for each evaluation, generating an index to classify the breathing predominant pattern of the individual, according to Wieler, et al. ${ }^{29}$ (2007).

\section{Dental Measurements}

In the study models, the measurements were made with the aid of a digital precision caliper (Mitutoyo, Suzano, SP, Brazil), keeping the

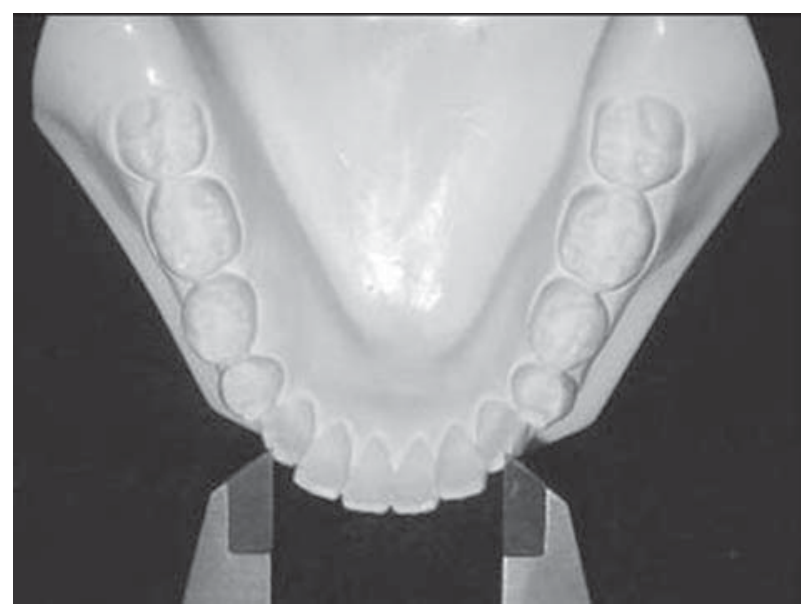

Figure 1- Measurement of intercanine distance pachymeter parallel to the occlusal face of the tooth. The mandibular intercanine distance from the tip of the cusp of the mandibular right canine to the tip of the left mandibular canine was measured in millimeters (Figure 1), as well as maxillary intercanine distance, using the same criteria, at moments 1 and 2 . Similarly, the mandibular intermolar distance from the tip of the mesiobuccal cusp of the right mandibular molar to the other molar on the left side (Figure 2), was quantified, applying the same methodology as used for the maxillary molars.

\section{Facial Measurements}

Cephalometric assessment was made by means of a combination of manual and computerized methods. The drawing of the anatomic structures was done manually, and after the tracing was digitized, the points were demarcated and the cephalometric values measured, using the cephalometric program RADIOCEF 2000 ${ }^{\circledR}$ (Radiomemory, Belo Horizonte, MG, Brazil). The study models were used to help to trace the dental relationships.

\section{The following measurements were used \\ Linear measurements with reference to the} pogonium: (Figure 3)

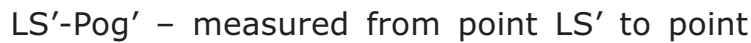
Pog', corresponding to the greatest anteroposterior dimension or distance of the lateral image of the mandibular symphysis (total thickness of the symphysis).

$\mathrm{LS}^{\prime}-\mathrm{B}^{\prime}$ - measured from point $\mathrm{LS}^{\prime}$ to point $\mathrm{B}^{\prime}$, corresponding to the greatest anteroposterior dimension or distance of the lateral image of the mandibular symphysis.

$B^{\prime}-$ Pog' $^{\prime}$ measured from point $B^{\prime}$ to point Pog', corresponding to the greatest anteroposterior dimension or distance of the lateral image of the mandibular symphysis.

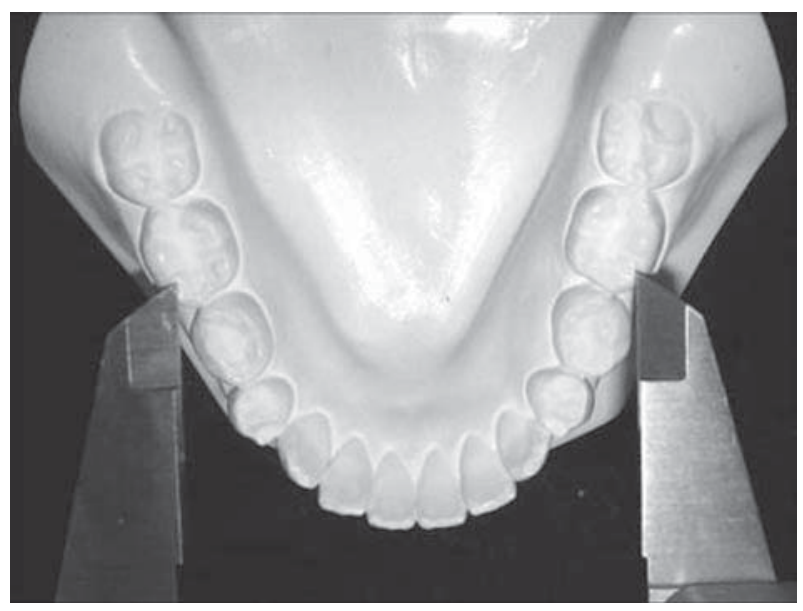

Figure 2- Measurement of intermolar distance 


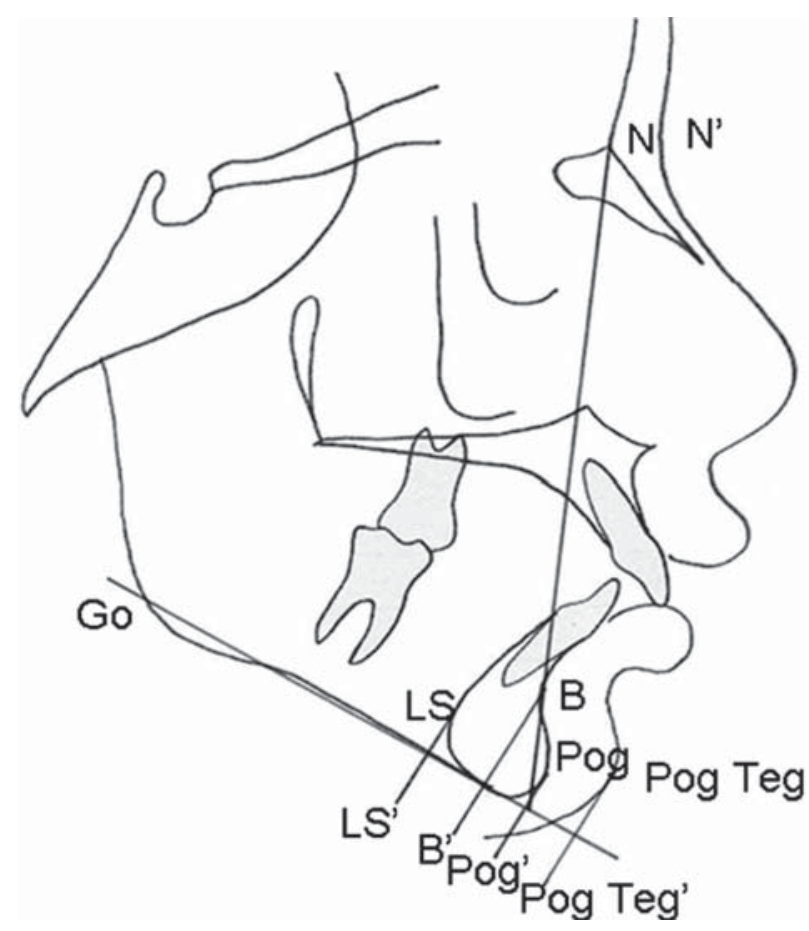

Figure 3- Pogonium cephalometric tracing with linear measurements

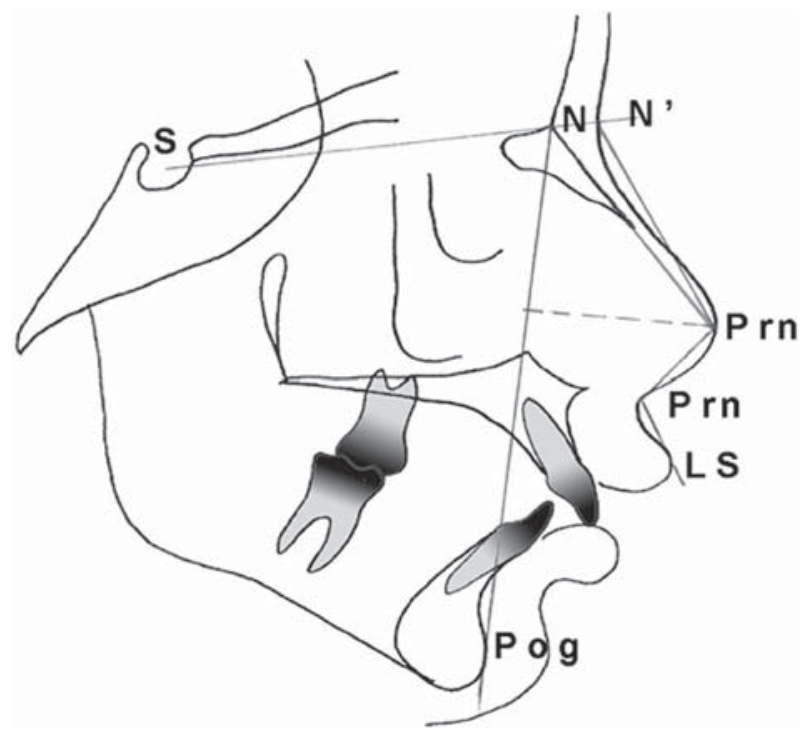

Figure 4- Nose cephalometric tracing with linear and angular measurements

Table 1- Comparison between intercanine and intermolar distances in nasal and mouth breathing according to the moments

\begin{tabular}{lcccc}
\hline Nasal Breathing & Mean (Moment 1 - 2) & Deviation & Std. Deviation & P \\
\hline Lower Intercanine 1 - Lower Intercanine 2 & $26.45-26.40$ & 0.05 & $0.81-0.90$ & 0.75 \\
Upper Intercanine1 - Upper Intercanine 2 & $34.82-34.61$ & 0.21 & $1.22-1.16$ & 0.40 \\
\hline Lower Intermolar 1 - Lower Intermolar 2 & $45.49-46.07$ & -0.58 & $1.14-1.28$ & $0.02^{*}$ \\
Upper Intermolar 1 - Upper Intermolar 2 & $51.36-51.95$ & -0.59 & $0.77-0.98$ & $0.00^{*}$ \\
\hline Mouth Breathing & & & & \\
Lower Intercanine 1 - Lower Intercanine 2 & $26.71-26.54$ & 0.17 & $0.75-0.59$ & 0.36 \\
\hline Upper Intercanine1 - Upper Intercanine 2 & $34.05-34.40$ & -0.35 & $0.62-0.75$ & $0.03^{*}$ \\
\hline Lower Intermolar 1 - Lower Intermolar 2 & $45.89-46.04$ & -0.15 & $1.02-1.53$ & 0.53 \\
\hline Upper Intermolar 1 - Upper Intermolar 2 & $50.99-51.05$ & -0.04 & $0.60-.87$ & 0.69 \\
\hline
\end{tabular}

Note: * $p<0.05$ indicates statistically difference

Table 2- Deviation comparison according to breathing mode

\begin{tabular}{lccccc}
\hline & Breathing Mode & N & Mean & Std. Deviation & P \\
\hline Lower Intercanine Deviation & Nasal & 24 & -0.21 & 3.10 & \\
& Mouth & 17 & -0.64 & 2.82 & 0.33 \\
\hline Upper Intercanine Deviation & Nasal & 24 & -0.68 & 3.62 & \\
\hline Lower Intermolar Deviation & Mouth & 17 & 1.08 & 1.83 & $0.04^{*}$ \\
\hline \multirow{2}{*}{ Upper Intermolar Deviation } & Nasal & 24 & 1.30 & 2.49 & \\
& Mouth & 17 & 0.34 & 2.29 & 0.11 \\
\hline
\end{tabular}

Note: * $p<0.05$ indicates statistically difference 
Pog'-PogTeg' - measured from point Pog' to point Pog Teg', corresponding to the greatest anteroposterior dimension or distance of the lateral image of the mandibular symphysis.

Line NB - union of points $N$ and $B$.

Pog-NB - distance from point Pog to line NB. Indicates the bone prominence in the most anterior region of the mentum as from line NB. Positive values when located in front of the line and negative when situated beyond line NB.

Linear measurements with reference to the nose: (Figure 4)

$\mathrm{N}^{\prime}-\mathrm{Prn}$ - measured from the tip of the nose in relation to the nasium. Determined by nose projection.

Prn-NPog - measured from the nasal depth in relation to the facial plane.

Angular measurements with reference to the nose:

SN-Prn-Sn - measured from the prominence of the nose in relation to the nasium - sella.

Prn-Sn-LS - nasium - labial angle - measured from the inclination of the columella in relation to the upper lip.

Table 3- Comparison between pogonium measurements in nasal and mouth breathing according to the moments

\begin{tabular}{lcccc}
\hline Nasal Breathing & Mean (Moment 1 - 2) & Deviation & Standard Deviation & P \\
\cline { 2 - 5 } LS-Pog 1 - LS-Pog 2 & $15.20-15.22$ & -0.02 & $2.36-1.68$ & 1.42 \\
\hline LS'-B' 1 - LS'-B' 2 & $10.08-8.96$ & 1.12 & $1.61-1.77$ & $0.00^{*}$ \\
\hline BPog 1 - BPog 2 & $6.53-6.19$ & 0.34 & $3.86-1.83$ & 0.39 \\
\hline Pog'-PogTeg' 1 - Pog'-PogTeg' 2 & $10.54-10.80$ & -0.26 & $2.31-2.33$ & 0.33 \\
\hline NB Line 1 - NB Line 2 & $95.95-100.03$ & -4.08 & $6.06-5.56$ & $0.00^{*}$ \\
\hline Pog-NB 1 - Pog-NB 2 & $1.85-2.43$ & -0.58 & $1.38-1.39$ & $0.01^{*}$ \\
\hline Mouth Breathing & & & & \\
\hline LS-Pog 1 - LS-Pog 2 & $14.71-15.10$ & -0.39 & $1.83-2.01$ & 0.35 \\
\hline LS'-B' 1 - LS'-B' 2 & $9.75-8.95$ & 0.80 & $1.80-2.02$ & $0.00^{*}$ \\
\hline BPog 1 - BPog 2 & $5.12-6.31$ & -1.19 & $1.36-1.66$ & 0.25 \\
\hline Pog'-PogTeg' 1 - Pog'-PogTeg' 2 & $10.40-10.98$ & -0.58 & $2.27-2.38$ & 0.05 \\
\hline NB Line 1 - NB Line 2 & $98.73-101.48$ & -2.75 & $4.67-4.73$ & $0.00^{*}$ \\
\hline Pog-NB 1 - Pog-NB 2 & $1.27-1.87$ & -0.50 & $1.22-1.33$ & $0.01^{*}$ \\
\hline
\end{tabular}

Note: * $p<0.05$ indicates statistically difference

Table 4- Comparison between pogonium measurements in moments according to breathing mode

\begin{tabular}{lcccc}
\hline Initial Moment (Moment 1) & Mean (Nasal and Mouth Breathing) & Deviation & Std. Deviation & P \\
\cline { 2 - 5 } LS-Pog Nasal - LS-Pog Mouth & $15.20-14.71$ & 0.49 & $2.36-1.83$ & 0.74 \\
\hline LS-B Nasal - LS-B Mouth & $10.08-9.75$ & 0.33 & $1.61-1.80$ & 0.97 \\
\hline BPog Nasal - BPog Mouth & $6.53-5.12$ & 1.41 & $3.86-1.36$ & 0.50 \\
\hline Pog'-PogTeg' Nasal - Pog'-PogTeg' Mouth & $10.54-10.40$ & 0.14 & $2.31-2.27$ & 0.89 \\
\hline NB Line Nasal - NB Line Mouth & $95.95-98.73$ & -2.78 & $6.06-4.67$ & 0.64 \\
\hline Pog-NB Nasal - Pog-NB Mouth & $1.85-1.27$ & 0.58 & $1.38-1.22$ & 0.84 \\
\hline Final Moment (Moment 2) & & & & \\
LS-Pog Nasal - LS-Pog Mouth & $15.22-15.10$ & 0.12 & $1.68-2.01$ & 0.63 \\
\hline LS-B Nasal - LS-B Mouth & $8.96-8.95$ & 0.01 & $1.77-2.02$ & 1.00 \\
\hline BPog Nasal - BPog Mouth & $6.19-6.31$ & -0.12 & $1.83-1.66$ & 0.68 \\
\hline Pog'-PogTeg' Nasal - Pog'-PogTeg' Mouth & $10.80-10.98$ & -0.18 & $2.33-2.38$ & 0.86 \\
\hline NB Line Nasal - NB Line Mouth & $100.03-101.48$ & -1.45 & $5.56-4.73$ & 0.92 \\
\hline Pog-NB Nasal - Pog-NB Mouth & $2.43-1.87$ & 0.56 & $1.39-1.33$ & 0.88 \\
\hline
\end{tabular}

Note: $p>0.05$ indicates statistically difference 
Table 5- Comparison between nose measurement in nasal and mouth breathing according to the moments

\begin{tabular}{lcccc}
\hline Nasal Breathing & Mean (Moment 1 - 2) & Deviation & Standard Deviation & P \\
\cline { 2 - 6 } NPRN 1 - NPRN 2 & $48.46-51.17$ & $-2,71$ & $1.55-1.95$ & $0.00^{*}$ \\
\hline PRNNPOG 1 - PRNNPOG 2 & $31.44-33.37$ & -1.93 & $3.05-1.66$ & 0.06 \\
\hline NPRNSN 1 - NPRNSN 2 & $99.74-96.09$ & 3.65 & $1.59-1.98$ & $0.00^{*}$ \\
PRNSNL 1 - PRNSNL 2 & $125.76-126.48$ & -0.72 & $3.90-4.08$ & $0.04^{*}$ \\
\hline Mouth Breathing & & & & \\
NPRN 1 - NPRN 2 & $48.65-51.09$ & $-2,44$ & $2.55-2.85$ & $0.00^{*}$ \\
\hline PRNNPOG 1 - PRNNPOG 2 & $31.16-33.54$ & -2.38 & $2.24-2.48$ & $0.04^{*}$ \\
NPRNSN 1 - NPRNSN 2 & $100.88-98.00$ & 2.88 & $4.24-3.89$ & $0.02^{*}$ \\
PRNSNL 1 - PRNSNL 2 & $120.59-122.76$ & -2.17 & $4.60-4.75$ & 0.14 \\
\hline
\end{tabular}

Note: * $p<0.05$ indicates statistically difference

Table 6- Comparison between nose measurement in moments according to nasal and mouth breathing

\begin{tabular}{lcccc}
\hline Initial Moment (Moment 1) & Mean (Nasal and Mouth Breathing) & Deviation & Std. Deviation & P \\
\cline { 2 - 5 } NPRN Nasal - NPRN Mouth & $48.46-48.65$ & $-0,19$ & $1.55-2.55$ & 1.00 \\
\hline PRNNPOG Nasal - PRNNPOG Mouth & $31.44-31.16$ & 0.28 & $3.05-2.24$ & 1.00 \\
\hline NPRNSN Nasal - NPRNSN Mouth & $99.74-100.88$ & -1.14 & $1.59-4.24$ & 0.96 \\
PRNSNL Nasal - PRNSNL Mouth & $125.76-120.59$ & 5.17 & $3.90-4.60$ & 0.43 \\
\hline Final Moment (Moment 2) & & & \\
NPRN Nasal - NPRN Mouth & $51.17-51.09$ & 0.08 & $1.95-2.85$ & 1.00 \\
\hline PRNNPOG Nasal - PRNNPOG Mouth & $33.37-33.54$ & -0.17 & $1.66-2.48$ & 1.00 \\
NPRNSN Nasal - NPRNSN Mouth & $96.09-98.00$ & -1.91 & $1.98-3.89$ & 0.85 \\
\hline PRNSNL Nasal - PRNSNL Mouth & $126.48-122.76$ & 3.72 & $4.08-4.75$ & 0.37 \\
\hline
\end{tabular}

Note: * $p<0.05$ indicates statistically difference

\section{RESULTS}

\section{Dental Measurements}

The Kolmogorov-Smirnov statistical test indicated that all the variables presented homogeneity of variances between the respiratory modes $(p<0.05)$. Therefore, moments 1 and 2 were compared to verify whether there were differences both for the nasal and mouth breathing modes, the Student's- $t$ test for paired samples was used. For the nose breathers, there was statistically significant increase $(p<0.05)$ in the intermolar distance between moments 1 and 2 for the mandible and maxilla (Table 1). For the mouth breathers, significant difference was observed $(p<0.05)$ only for the maxillary intercanine distance, there being an increase from moment 1 to moment 2 (Table 1 ). In the comparison between the variations from moment 1 to moment 2, it was observed that there was statistically significant difference according to the respiratory mode, and for the analysis, the Student's- $t$ test for independent samples was used. The result of the test indicated that there was statistically significant difference $(p<0.05)$ for the variable maxillary intercanine distance, with the group of mouth breathers presenting a positive and larger variation than the group of nose breathers, which presented negative variation. With regard to the variable maxillary intermolar distance, there was a significant difference $(p<0.05)$, with the group of mouth breathers presenting a positive variation, smaller than that of the group of nose breathers (Table 2).

\section{Facial Measurements \\ Pogonium}

Since the Kolmogorov-Smirnov statistical test indicated that all the variables, in all the moments presented normal distribution, with the exception of B'-Pog' and Pog-NB, comparison of the mean values according to moments was performed by two-way ANOVA with repeated measures and Tukey HSD multiple comparison test.

The results of this test demonstrated that

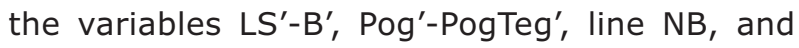
Pog-NB presented significant difference $(p<0.05)$ between the initial and final moments, without any interference of the respiratory mode. The variables 
$B^{\prime}$-Pog' and LS'-PogTeg' presented no significant difference $(p>0.05)$ (Table 3 and 4$)$.

\section{Nose}

Since the Kolmogorov-Smirnov statistical test indicated that all the variables, with the exception of Prn-NPog, in all the moments presented normal distribution, comparison of the mean values according to moments was performed by two-way ANOVA with repeated measures and Tukey HSD multiple comparison test.

The results of this test showed that the variables presented statistically significant difference $(p<0.05)$ between the initial and final moments, without any interference of the breathing mode (Tables 5 and 6).

\section{DISCUSSION}

Respiratory function and its influence on the development of orofacial structures has been a subject of debates in articles, texts and researches for over a century. Various studies have investigated the possible repercussions of the respiratory pattern on the functional, neuromuscular, skeletal and dental functions ${ }^{13,14,18,28}$.

Nasal respiration is primordial in order for correct growth and development of the craniofacial complex to occur ${ }^{10,20}$, but some authors that disagree that facial morphology and the respiratory mode are intimately related ${ }^{14,18,28}$. According to Klein ${ }^{13}(1986)$, there is no conclusive evidence that obstruction of nasal respiration alters facial growth and development.

Harvold, et al..$^{12}$ (1981), in a study with animals, observed that after 9 and 15 months of eminently mouth breathing, the maxillary intercanine distance was significantly smaller in the monkeys with obstructed nostrils in comparison with the control animals. The mandibular intercanine distance was also significantly smaller in the experimental monkeys.

In this study, there was an increase in the dental distances assessed in nose and mouth breather individuals, except for the variable maxillary intercanine distance in nose breathers, in which there was narrowing, as demonstrated by Carter and McNamara Júnior ${ }^{5}$ (1998). On the other hand, Trask and Shapiro ${ }^{26}$ (1987) found no differences in the intercanine and intermolar widths between mouth and nose breathers.

The importance of maintaining dental arch shape and the intercanine and intermolar distances in the maxilla, and particularly, the intercanine distance in the mandible is described in the literature over the course of time, as it is a fact that teeth moved by orthodontic appliances tend to revert to their original positions ${ }^{3,23}$. For some authors, moderate increases in these distances can be tolerated, while for others, any change, even if minor, may cause instability.

The age range in this study was from 10 and 16 years, differing from Formby, et al. ${ }^{9}$ (1994), who studied the range between 18 and 42 years and observed significant differences in the growth of hard and soft tissues in both genders, especially, an increase in all the dimensions of the nose.

Tourne and Schweiger ${ }^{25}$ (1996) found differences in the craniofacial development between mouth and nose breathers, with predominantly mouth breathers presenting greater inclination of the mandibular plane angle and a vertical growth pattern.

In the present research, there was alteration in the skeletal pogonium and tegumentary measurements according to the moments, in agreement with $\operatorname{Koch}^{16}$ (1979).

Increase in the measurement LS'-Pog' was observed, as a result of differentiated bone remodeling with greater bone apposition in the lingual region of the pogonium, resulting in an increase in the tegumentary pogonium (Pog'PogTeg'). These changes characterize skeletal growth, as proved by the histological studies of Enlow and Harris ${ }^{7}$ (1964) and the metal implants of Bjork² (1963).

In nose breathers, a decrease in the measurement $B^{\prime}$-Pog' was verified, and in mouth breathers there was an increase. Longitudinal studies by Bjork ${ }^{2}$ (1963) and Enlow and Harris ${ }^{7}$ (1964) proved that the thickening of the symphysis is due to the greater bone apposition in the lingual region, while the region of the pogonium is more stable and grows very little or not at all. However, in this study, it was not possible to assess the decrease ratio in $\mathrm{B}^{\prime}$ Pog'. One supposes that the decrease in $B^{\prime}-$ Pog $^{\prime}$ and

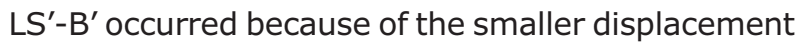
in the posterior direction of point $B$, as a result of downward and backward rotation of the mandible, not ignoring the possibility of less appositional growth in the region of the pogonium.

Significant increase in the measurement PogNB was noted, but it depended significantly on the position of point $B$, due to the combination of displacement towards the posterior direction of point $B$ and bone apposition in the pogonium, in addition to variations in the inclination of the mandibular plane angle.

With regard to analysis of the nose, there was significant increase $(p \leq 0.05)$ in the nasal length and depth, measured by means of the values of $\mathrm{N}^{\prime}$-Prn and angle Prn-NPog, at the initial and final moments, without influence of the respiratory mode.

The linear variable N-Prn-Sn, corresponding to nasal projection in the anteroposterior plane, presented statistically significant alteration in the 
present study. These data are in agreement with those of Brant, et al. ${ }^{4}$ (2006), who demonstrated significant changes in N-Prn-Sn when they compared the groups with and without extraction of premolars.

The nasolabial angle (Prn-Sn-LS) revealed significant modifications $(p \leq 0.05)$ between the moments, however, without presenting interaction with the respiratory mode. The data are in agreement with Salgado ${ }^{22}$ (2003), who observed that significant variations occur in the nasolabial angle in individuals with Angle's Class II and III malocclusions, the values in Class II being higher.

The interrelation between the tegumentary tissue of the nose and pogonium, as well as the dental alterations must be considered in the diagnosis, when establishing the plan and performing the treatment in individuals in the growth phase, particularly in patients with predominantly mouth breathing.

\section{CONCLUSIONS}

There was change in the nose and skeletal and tegumentary pogonium in the anteroposterior plane, during the course of growth, without interference of the breathing pattern. On the other hand, the maxillary and mandibular intercanine and intermolar distances presented significant differences between the moments and breathing pattern.

\section{REFERENCES}

1- Aksu M, Kocadereli I. Arch width changes in extraction and nonextraction treatment in Class I patients. Angle Orthod. 2005;75(6):948-52.

2- Bjork A. Variations in the growth pattern of the human mandible: longitudinal radiographic study by the implant method. J Dent Res. 1963;42(1):400-11.

3- Boley JC, Mark JA, Sachdeva RC, Buschang PH. Long-term stability of Class I premolar extraction treatment. Am J Orthod Dentofacial Orthop. 2003;124(3):277-87.

4- Brant JCO, Siqueira VCV. Soft tissue changes evaluated in Class II, division 1 cases, after orthodontic treatment. Rev Dent Press Ortodon Ortopedi Facial. 2006;11(2):93-102.

5- Carter GA, McNamara JA Jr. Longitudinal dental arch changes in adults. Am J Orthod Dentofacial Orthop. 1998;114(1):88-99.

6- Chaconas SJ. A statistical evaluation of nasal growth. Am J Orthod. 1969;56(4):403-14.

7- Enlow DH, Harris DB. A study of the postnatal growth of the human mandible. Am J Orthod. 1964;50(1):25-50.
8- Erdinc AE, Nanda RS, Işiksal E. Relapse of anterior crowding in patients treated with extraction and nonextraction of premolars. Am J Orthod Dentofacial Orthop. 2006;129(6):775-84

9- Formby WA, Nanda RS, Currier GF. Longitudinal changes in the adult facial profile. Am J Orthod Dentofacial Orthop. 1994;105(5):464-76.

10- Graber TM, Vanarsdall RL Jr. Orthodontics: current principles and techniques. $3^{\text {rd }}$ ed. St. Louis: Mosby; 2000.

11- Hambleton RS. The soft tissue covering of the skeletal face as related to orthodontic problems. Am J Orthod. 1964;50(6):405-20. 12- Harvold EP, Tomer BS, Vargervik K, Chierici G. Primate experiments on oral respiration. Am J Orthod. $1981 ; 79(4): 359-72$. 13- Klein JC. Nasal respiratory function and craniofacial growth. Arch Otolaryngol Head Neck Surg. 1986;112(8):843-9.

14- Kluemper GT, Vig PS, Vig KW. Nasorespiratory characteristics and craniofacial morphology. Eur J Orthod 1995;17(6):491-5.

15- Knott VB. Longitudinal study of dental arch widths at four stages of dentition. Angle Orthod. 1972;42(4):387-94.

16- Koch R, Gonzales A, Witt E. Profile and soft tissue changes during and after orthodontic treatment. Eur J Orthod. 1979;1(3):193-9.

17- Lee RT. Arch width and form: a review. Am J Orthod Dentofacial Orthop. 1999;115(3):305-13.

18- Nowak AJ, Warren JJ. Infant oral health and oral habits. Pediatr Clin North Am. 2000;47(5):1043-66.

19- Prahl-Andersen B, Ligthelm-Bakker AS, Wattel E, Nanda R. Adolescent growth changes in soft tissue profile. Am J Orthod Dentofacial Orthop. 1995;107(5):476-83.

20- Proffit WR, Fields HW, Sarver DM. Contemporary orthodontics. $4^{\text {th }}$ ed. St. Louis: Mosby; 2007.

21- Ricketts RM. Planning treatment on the basis of the facial pattern and an estimate of its growth. Angle Orthod. 1957;27(1):14-37.

22- Salgado JAP, Moraes LC, Castilho JCM, Moraes MEL. Nasolabial angle evaluation, in lateral cephalometric $x$-rays, divided in superior and inferior angle, by a parallel line to the Frankfort plane, in individuals bearers of Class II and Class III occlusion. Cienc Odontol Bras. 2003;6(3):40-9.

23- Sinclair PM, Little RM. Maturation of untreated normal occlusions. Am J Orthod. 1983;83(2):114-23.

24- Steiner CC. Cephalometrics in clinical practice. Angle Orthod. $1959 ; 29(1): 8-29$.

25- Tourne LP, Schweiger J. Immediate postural responses to total nasal obstruction. Am J Orthod Dentofacial Orthop. $1996 ; 110(6): 606-11$.

26- Trask GM, Shapiro GG, Shapiro PA. The effect of perennial allergic rhinitis on dental skeletal development: a comparison of sibling pairs. Am J Orthod Dentofacial Orthop. 1987;92(4):286-93. 27- Ward DE, Workman J, Brown R, Richmond S. Changes in arch width. A 20-year longitudinal study of orthodontic treatment. Angle Orthod. 2006;76(1):6-13.

28- Warren DW. Effect of airway obstruction upon facial growth. Otolaryngol Clin North Am. 1990;23(4):699-712.

29- Wieler WJ, Barros AM, Barros LA, Camargo ES, Ignácio SA, Maruo $\mathrm{H}$, et al. A combined protocol to aid diagnosis of breathing mode. Rev Clin Pesq Odontol. 2007;3(2):101-14. 